\title{
On Open Charm Production in Heavy Ion Collisions
}

\author{
F.O. Durães ${ }^{1,2}$, F.S. Navarra ${ }^{2}$, and M. Nielsen ${ }^{2}$ \\ ${ }^{1}$ Departamento de Física, Faculdade de Ciências Biológicas, Exatas e Experimentais, \\ Universidade Presbiteriana Mackenzie, C.P. 01302-907 São Paulo, Brazil \\ ${ }^{2}$ Instituto de Física, Universidade de São Paulo, C.P. 66318, 05389-970 São Paulo, SP, Brazil
}

Received on 15 August, 2003.

\begin{abstract}
We update the calculation of $c-\bar{c}$ production in the initial stage of nucleus - nucleus collisions and within quark gluon plasma, using the most recent and most accepted values for the ingredients, such as nuclear parton distributions, quark masses, couplings and fireball parameters. We find a large discrepancy in the charm yields, depending on the input choices. A global analysis of all the different cases suggests that the "in-plasma" production is a significant fraction of the total yield.
\end{abstract}

Open charm production in proton-proton collisions seems to be well understood in terms of perturbative QCD (pQCD)[1]. In nucleus-nucleus collisions, the presence of nuclear effects in the initial parton distributions introduces some uncertainties[2]. In view of the forthcoming PHENIX data on charm production in $A u+A u$ collisions at RHIC, it is necessary to clarify all the aspects of charm production. In this work we perform again some well known calculations and concentrate on $c \bar{c}$ production within the plasma. This is usually neglected because the number of $c \bar{c}$ pairs produced in the plasma is believed to be small. However, as it will be discussed, a closer look into the existing estimates shows discrepancies of two orders of magnitude. In [3], for example, it was estimated to be of the order of $1 \%$ of the total number of charm quark pairs. In [4], with the inclusion of thermal parton masses this fraction was estimated to reach $20-30 \%$. Finally, in [5] this number could, in some cases, be equal to the number of directly produced pairs. No systematic effort was made to reconcile these different estimates.

In leading order (LO) the cross section is computed with the use of perturbative QCD for the diagrams of the elementary processes $q \bar{q} \rightarrow c \bar{c}$ and $g g \rightarrow c \bar{c}$ convoluted with the parton densities in the projectile and in the target. Calling $x_{F}$ the fractional momentum of the produced pair (with respect to the momentum of a projectile nucleon in $\mathrm{cm}$ frame) and $\sqrt{s}$, the cm energy of a nucleon-nucleon collision, the cross section for inclusive $c-\bar{c}$ production is given by:

$$
\begin{aligned}
& \frac{d \sigma^{A B \rightarrow c \bar{c}}}{d x_{F}}=\kappa \int_{4 m_{c}^{2}}^{\infty} d m^{2} \\
& \times \frac{1}{s \sqrt{x_{F}^{2}+4 m^{2} / s}} H_{A B}\left(x_{1}, x_{2} ; m^{2}\right)
\end{aligned}
$$

where $x_{1,2}=\frac{1}{2}\left( \pm x_{F}+\sqrt{x_{F}^{2}+4 m^{2} / s}\right)$ and

$$
\begin{aligned}
& H_{A B}\left(x_{1}, x_{2} ; m^{2}\right)=A B\left\{f_{g} R_{g}^{A} f_{g} R_{g}^{B} \hat{\sigma}_{g g}\right. \\
& \left.+\left[f_{q} R_{q}^{A} f_{\bar{q}} R_{\bar{q}}^{B}+f_{\bar{q}} R_{\bar{q}}^{A} f_{q} R_{q}^{B}\right] \hat{\sigma}_{q \bar{q}}\right\}
\end{aligned}
$$

In the above expression $\hat{\sigma}_{g g}$ and $\hat{\sigma}_{q \bar{q}}$ are the elementary LO cross sections for the processes $g g \rightarrow c \bar{c}$ and $q \bar{q} \rightarrow c \bar{c}$ respectively, a sum over the flavor label $q$ is understood $(q=u, d, s)$ and $R_{i}^{A}\left(x, m^{2}\right)=\frac{f_{i}^{A}\left(x, m^{2}\right)}{f_{i}\left(x, m^{2}\right)}$, with $f_{i}^{A}\left(x, m^{2}\right)$ $\left(f_{i}\left(x, m^{2}\right)\right)$ being the $i$ parton momentum distribution in a nucleon inside the nucleus $A$ (free space). A factor $\kappa$ was introduced in (1) to account for higher order corrections. We write the above cross section in a differential form as a function of the variable $x_{F}$ in order to have a better control over the validity domain of our calculations. It is known that pQCD should not hold for large values of $x_{F}$ [6].

In a central $A+A$ collision the number of $c-\bar{c}$ pairs initially produced is related to the cross section by [7]:

$$
N_{Q C D}^{c \bar{c}}=\frac{1}{\pi R_{A}^{2}} \int_{0}^{1} d x_{F} \frac{d \sigma^{A A \rightarrow c \bar{c}}}{d x_{F}}
$$

where $R_{A}$ is the radius of nucleus $A$, given by $R_{A}=$ $\left(1.18 A^{1 / 3}-0.45\right) \mathrm{fm}$.

In Table I we present our results for the total number of initially produced charm quark pairs for different charm quark masses, with and without shadowing effects. The nuclear parton distribution functions are taken from Eskola $e t$ al. [8] and from Hirai et al. [9].

These numbers suggest that the commonly used value $N_{Q C D}^{c \bar{c}}=10$ is probably too large.

The computation of the in-plasma $c \bar{c}$ pair production rate goes back to the late eighties [10], was discussed in short papers (for example [3]), included in comprehensive review articles [11] during the nineties and experienced improvements due to advances in thermal field theory [4]. 
Assuming that QGP is formed, we then have a gas of quarks and gluons with momenta obeying respectively Fermi-Dirac and Bose-Einstein distributions that can collide producing $c \bar{c}$ pairs.

The charm production rate in the reaction $g g \rightarrow c \bar{c}$, at temperature $T$ is given by [12]:

$$
\begin{aligned}
& \frac{d N^{g g \rightarrow c \bar{c}}}{d t d^{3} x}=\frac{g_{g}^{2}}{2(2 \pi)^{6}} \kappa \\
& \times \int d^{3} p_{1} d^{3} p_{2} f_{g}\left(E_{1}\right) f_{g}\left(E_{2}\right) \hat{\sigma}_{g g}^{L O} v_{12}
\end{aligned}
$$

where $g_{g}$ is the gluon statistical factor (number of colors $\times$ number polarization states) $v_{12}$ is the relative velocity between colliding gluons with energies $E_{1}$ and $E_{2}$ and three momenta $\overrightarrow{p_{1}}$ and $\overrightarrow{p_{2}}, \hat{\sigma}_{g g}^{L O}$ is the elementary gluon-gluon cross section and $f_{g}\left(E_{i}\right)$ the usual thermal distribution function:

$$
f_{g}\left(E_{i}\right)=\frac{1}{e^{E_{i} / T}-1}
$$

A similar expression can be written for the process $q \bar{q} \rightarrow c \bar{c}$.

In order to account for expansion effects we shall assume that the system cools down following Bjorken hydrodynamics, in which the volume of the system evolves in time according to:

$$
V(\tau)=V_{0} \frac{\tau}{\tau_{0}} \quad \Longrightarrow \quad V(T)=V_{0}\left(\frac{T_{0}}{T}\right)^{3}
$$

where $V_{0}=\pi R_{A}^{2} \tau_{0}, T_{0}$ is the initial temperature and $\tau_{0}$ is the thermalization time, which marks the beginning of the hydrodynamical expansion $\left(\tau_{0}=0.7 \mathrm{fm}\right)$.

Expression (4) can thus be rewritten as:

$$
\begin{gathered}
\frac{d N^{g g \rightarrow c \bar{c}}}{d x_{F}}=\frac{3 \pi \sqrt{s} g_{g}^{2} \kappa}{8(2 \pi)^{6}} \int_{4 m_{c}^{2}}^{\infty} d m^{2} \int_{0}^{\infty} d p_{T}^{2} \int_{0}^{2 \pi} d \beta \\
\times \int_{T_{f}}^{T_{0}} \tau_{0} T_{0}^{3} T^{-4} V(T) d T \int_{0}^{\infty} d E_{1} f_{g}\left(E_{1}\right) f_{g}\left(E-E_{1}\right) \\
\times\left[\frac{1}{\left|H\left(h_{1}\right)\right|} \theta\left(E_{1}^{2}-h_{1}^{2}\right)+\frac{1}{\left|H\left(h_{2}\right)\right|} \theta\left(E_{1}^{2}-h_{2}^{2}\right)\right] \\
\times \hat{\sigma}_{g g}^{L O}\left(m^{2}\right) \frac{m^{2}}{E} \theta\left(E_{1}\right) \theta\left(E-E_{1}\right)
\end{gathered}
$$

where $E=\sqrt{m^{2}+p_{T}^{2}+p_{z}^{2}}$ is the energy of the $c \bar{c}$ pair of mass $m$ and:

$$
H\left(h_{1,2}\right)=2 p_{z}-\frac{2 p_{T} \cos (\beta)}{\left(E_{1}^{2}-h_{1,2}^{2}\right)^{1 / 2}} h_{1,2}
$$

and also

$$
\begin{gathered}
h_{1,2}=\frac{1}{2} \frac{1}{p_{z}^{2}+p_{T}^{2} \cos ^{2}(\beta)} \\
\times\left\{p _ { z } ( 2 E E _ { 1 } - m ^ { 2 } ) \mp \left\{p_{T}^{2} \cos ^{2}(\beta)\right.\right.
\end{gathered}
$$

$$
\left.\left.\times\left[4 E_{1}^{2}\left(p_{T}^{2} \cos ^{2}(\beta)+p_{z}^{2}\right)-\left(m^{2}-2 E E_{1}\right)^{2}\right]\right\}^{1 / 2}\right\}
$$

Including the differential rate due to the process $q \bar{q} \rightarrow c \bar{c}$ and integrating over $x_{F}$, we arrive at the total number of thermal $c \bar{c}$ pairs:

$$
N_{Q G P}^{c \bar{c}}=\int_{0}^{1} d x_{F}\left[\frac{d N^{g g \rightarrow c \bar{c}}}{d x_{F}}+\frac{d N^{q \bar{q} \rightarrow c \bar{c}}}{d x_{F}}\right]
$$

There are some known papers on the subject $[3,4,5$, 11, 13]. From the reading of these papers, we conclude that there are large discrepancies in the numbers and in the way to obtain them. The sources of these discrepancies are: a) initial temperature of the plasma, $T_{0}$ (ranging from $300 \mathrm{MeV}$ to $550 \mathrm{MeV}$ ); b) degree of parton equilibration (described by the fugacity factors); c) initial volume and/or thermalization time, $V_{0}$ and $\tau_{0}$; d) total energy contained in the fireball; e) use or not of a $\kappa$ factor (=2) in computing thermal rates; f) use or not of temperature dependent $\alpha_{s} ; \mathbf{g}$ ) mass of the charm quark $m_{c}$ (going from 1.2 to $1.5 \mathrm{GeV}$ ); h) use or not of thermal masses for gluons and quarks in the reactions $g+g \rightarrow c+\bar{c}$ and $q+\bar{q} \rightarrow c+\bar{c}$.

Depending on the choices that one has to make in dealing with a) $\rightarrow$ h) the final value of in-plasma produced $c \bar{c}$ pairs can change by orders of magnitude, going roughly from $N_{Q G P}^{c \bar{c}}=0.02$ in [3] to up 15 in [11]. In a comprehensive analysis this same variation was found in [5]. In [4], the authors arrive to the conclusion that in-plasma production was only a factor two smaller than the initial production. However in that paper the factor $\kappa=2$ was not included in calculation. If it were, then $N_{Q G P}^{c \bar{c}} \simeq N_{Q C D}^{c \bar{c}}$.

We have explicitly investigated the effect of changing the mass of the quark $c$ and the differences which arise when we use the coupling constant running with $m^{2}$ or with $T$ [4]:

$$
\begin{gathered}
\alpha_{s}\left(m^{2}\right)=\frac{12 \pi}{\left(33-2 N_{F}\right) \ln \frac{m^{2}}{\Lambda_{Q C D}^{2}}} \\
\alpha_{s}(T)=\frac{6 \pi}{\left(33-2 N_{F}\right) \ln \frac{19 T}{\Lambda_{\overline{M S}}}}
\end{gathered}
$$

where $N_{F}=3, \Lambda_{Q C D}=230 \mathrm{MeV}$ and $\Lambda_{\overline{M S}}=80 \mathrm{MeV}$.

In Table II we present our results for the number of "inplasma born" $c \bar{c}$ pairs for different values of couplings and charm quark masses. All the calculations were done with an initial plasma temperature of $T_{0}=550 \mathrm{MeV}, \alpha_{s}\left(M^{2}\right)$ and $\alpha_{s}(T)$ given by (8) and (9) respectively. The numbers inside parenthesis correspond to the choice $\kappa=1$. Otherwise the numbers were obtained with $\kappa=2$.

The fourth line of Table II shows that our results may change by a factor 30 depending on the inputs used. In some cases a direct comparison with other works is possible. For example, in the second column of Table II, comparing the numbers in parenthesis, we notice that we obtain nearly five 
TABLE 1. Number of $c \bar{c}$ pairs produced in $A u-A u$ collisions at RHIC from QCD calculations for different values of couplings and charm quark masses. The nuclear parton distribution functions are taken from Eskola et al. (EKS) [8] and from Hirai et al. (HKM) [9].

\begin{tabular}{rrrrr}
\hline & \multicolumn{2}{c}{$m_{c}=1.2 \mathrm{GeV}$} & \multicolumn{2}{c}{$m_{c}=1.5 \mathrm{GeV}$} \\
& EKS & HKM & EKS & HKM \\
\hline$N_{Q C D}^{c \bar{c}}$ (without shadowing) & 6.52 & 4.65 & 2.65 & 2.07 \\
(with shadowing) & 6.32 & 4.36 & 2.61 & 1.97 \\
\hline
\end{tabular}

TABLE 2. Number of "in-plasma" produced $c \bar{c}$ pairs in RHIC collisions. Numbers inside parenthesis are obtained with $\kappa=1$ and the others with $\kappa=2$. The initial temperature is $550 \mathrm{MeV}$ in all cases.

\begin{tabular}{|c|c|c|c|c|c|}
\hline & \multirow[t]{2}{*}{ QGP } & \multicolumn{2}{|c|}{$m_{c}=1.2 \mathrm{GeV}$} & \multicolumn{2}{|c|}{$m_{c}=1.5 \mathrm{GeV}$} \\
\hline & & $\alpha_{s}\left(M^{2}\right)$ & $\alpha_{s}(T)$ & $\alpha_{s}\left(M^{2}\right)$ & $\alpha_{s}(T)$ \\
\hline \multirow{4}{*}{$N_{Q G P}^{c \bar{c}}$} & Levai el al. [4] & $-\quad-$ & $-(3.7)$ & $-\quad-$ & $-(1.1)$ \\
\hline & Rafelski el al. [11] & - & - & $-(15)$ & \\
\hline & Müller el al. [5] & & - & $17-$ & \\
\hline & This work & $120(60)$ & $39(19.5)$ & $22(11)$ & $7.6(3.8)$ \\
\hline
\end{tabular}

times more pairs than in [4]. A similar excess is observed comparing the numbers in parenthesis in the fourth column. Comparing our work with Ref. [4] we can see that the pair production mechanism is quite similar but the treatment given to the plasma expansion is different. Whereas we have used the standard Bjorken hydrodynamics, in Ref. [4] a new hydrodynamical model was introduced. Comparing the details of both approaches we concluded that in Ref. [4] the expansion and cooling of the system is much faster than in Bjorken hydrodynamics. Consequently the system stops much earlier to create $c \bar{c}$ pairs and the final yield will be smaller. In order to remove this discrepancy, the use of a state-ofthe-art hydrodynamical code is required. This is hard work still to be done.

The comparison of the other results in Table II with other works shows that for similar inputs we obtain numbers which are compatible with those presented in Refs. [5] and [11]. The results both for $N_{Q C D}^{c \bar{c}}$ and for $N_{Q G P}^{c \bar{c}}$ do not include final state interactions or hadronization effects, which might change them slightly [14].

Finally, it should be mentioned that in some previous works (see, for example, [5]) small parton fugacity factors have been used and this reduced considerably the final number of thermal $c \bar{c}$ pairs. In view of the recent measurements of anisotropic flow in $A u+A u$ collisions at RHIC, which point to very rapid formation of equilibrated hot matter [15], the use of such small factors seems unjustified.

In this note we present an update of charm yields in nucleus nucleus collisions. Although it is difficult to draw firm conclusions at this stage, it seems that the "in-plasma" production is a significant fraction of the total yield and can not be neglected as it was in the recent works on the subject. Moreover, if this yield is large there will be higher chances of producing Coulomb bound states, as discussed in [16].

\section{Acknowledgements}

This work has been supported by CNPq and FAPESP. We are indebted to S. Narison, X.N. Wang and H. Satz for usefull comments.

\section{References}

[1] R. Vogt, hep-ph/0203151 and references therein.

[2] R. Vogt, Phys. Rept. 310, 197 (1999).

[3] B. Müller and X.N. Wang, Phys. Rev. Lett. 68, 2437 (1992).

[4] R. Vogt and P. Levai, Phys. Rev. C56, 2707 (1997).

[5] P. Levai, B. Müller and X-N. Wang, Phys. Rev. C 51, 3326 (1995).

[6] F. Carvalho, F.O. Durães, F.S. Navarra and M. Nielsen, Phys. Rev. Lett. 86, 5434 (2001); F.O.Durães, F.S.Navarra, C. A. A. Nunes and G.Wilk, Phys. Rev. D53, 6136 (1996) .

[7] K. J. Eskola, K. Kajantie and J. Lindfors Nucl. Phys. B 323, 37 (1989).

[8] K. J. Eskola, V. J. Kolhinen and C. A. Salgado, Eur. Phys. J. C9, 61 (1999).

[9] M. Hirai, S. Kumano and M. Miyama, Phys. Rev. D64, 034003 (2001).

[10] A. Shor, Phys. Lett. B215, 375 (1988).

[11] J. Rafelski, J. Letessier, and A. Tounsi, Acta Phys. Pol. B27, 1037 (1996).

[12] "Introduction to High-Energy Heavy-Ion Collisions, CheukYin Wong, World Scientific Publishing Co. Pte. Ltd, 1994.

[13] M. Schroedter, R.L. Thews, and J. Rafelski, J. Phys. G27, 691 (2001).

[14] For recent developments see: F.O. Durães, Hung-chong Kim, Su Houng Lee, F.S. Navarra and M. Nielsen, Phys. Rev. C68, 035208 (2003); F.O. Durães, Su Houng Lee, F.S. Navarra and M. Nielsen, Phys. Lett. B564, 97 (2003); F.S. Navarra, M. Nielsen and M.R. Robilotta, Phys. Rev. C64, 021901 (R) (2001); F.S. Navarra, M. Nielsen, R.S. Marques de Carvalho and G. Krein, Phys. Lett. B529, 87 (2002).

Phys. Rev. C68, 035208 (2003)

[15] B. Mueller and A. Schaefer, hep-ph/0306309 and references therein.

[16] F.O. Durães, F.S. Navarra and M. Nielsen, Phys. Rev. C68, 044904 (2003). 